\title{
SEPARAÇÃO SÓLIDO-FLUIDO NO CAMPO GRAVITACIONAL: GERAÇÃO DE MATERIAL DIDÁTICO EMPREGANDO SIMULAÇÕES CFD
}

\author{
N. P. ALMEIDA ${ }^{1}$, M. C. CANHADAS ${ }^{1}$, J. L. V. NETO ${ }^{1}$ e K. G. dos $\operatorname{SANTOS}^{1}$ \\ ${ }^{1}$ Universidade Federal do Triângulo Mineiro, Departamento de Engenharia Química \\ E-mail para contato: kassiagsantos@gmail.com
}

\begin{abstract}
RESUMO - O crescimento da economia brasileira acarretou no aumento da demanda de engenheiros e na expansão das Universidades Brasileiras. Quanto ao ensino de Operações Unitárias (OP), observa-se uma escassez de material didático interativo e ilustrativo. As ferramentas de modelagem e simulação são uma excelente alternativa para a predição da fluidodinâmica de fluidos e partículas e têm sido utilizadas no projeto e otimização de equipamentos da indústria, fornecendo informações do processo em locais de difícil aquisição de medidas experimentais. Este trabalho apresenta a simulação por Fluidodinâmica Computacional (CFD) de um dos exercícios apresentados em sala de aula sobre separação sólido-fluido no campo gravitacional. Foram avaliados o efeito do tamanho, forma e densidade das partículas sobre a eficiência de coleta. As figuras e vídeos gerados neste trabalho facilitarão a compreensão dos alunos quanto aos fenômenos envolvidos na coleta de partículas dentro da câmara de separação.
\end{abstract}

\section{INTRODUÇÃO}

A rápida evolução da indústria, juntamente com o aparecimento de novas ferramentas pedagógicas e científicas, representa um formidável desafio para o ensino da Engenharia, principalmente na área de projeto e otimização de equipamentos.

O entendimento de Operações Unitárias é muito importante na formação de um engenheiro químico. No caso do projeto de equipamentos de separação de sólido-fluido, ministrado nas disciplinas de Operações Unitárias, os alunos têm apresentado dificuldades de compreensão do funcionamento dos equipamentos e muitas vezes demonstram ausência de conceitos físicos sobre o escoamento de fluidos e partículas. Observa-se também a dificuldade dos alunos em estabelecer estas correspondências entre os esquemas dos equipamentos e as imagens reais e principalmente de visualizá-las tridimensionalmente. Um dos maiores problemas encontrados na etapa de preparo das aulas é a escassez de imagens do interior dos equipamentos e imagens dos mesmos em funcionamento. A eficiência de um equipamento na coleta de uma partícula, por exemplo, pode ser explicada pela fluidodinâmica, que mostra as interações entre o fluido e a partícula dentro de uma determinada geometria. Ao visualizar essa geometria, e a posição das partículas dentro do equipamento, o aluno passa a entender as diferenças básicas entre os equipamentos e o porquê deles terem eficiências de coleta diferentes.

Uma forma de minimizar este problema é modificar a forma de apresentação destes conteúdos, através da informática, tornando o ambiente em sala de aula mais atrativo. Neste 
sentido, imagens e vídeos que mostrem aos alunos o que se passa no interior dos equipamentos durante sua operação seria uma ferramenta didática poderosa. Os softwares de simulação computacional aparecem como uma opção interessante na predição da fluidodinâmica do fluido e do comportamento das partículas que escoam através de vários equipamentos encontrados em processos químicos. Esses softwares de CFD são resolvedores de sistemas das equações de transporte, que empregam métodos numéricos para obter campos de velocidades, temperatura e etc., possibilitando assim avaliar o transporte de uma propriedade de interesse e gerando resultados com alta capacidade gráfica (Santos, 2011).

A técnica de Fluidodinâmica Computacional tem se destacado em aplicações de engenharia, como na mecânica dos fluidos. Pacotes comerciais com modelos clássicos, que estabelecem relações para as principais variáveis relacionadas ao escoamento de fluidos e o transporte de calor e massa, têm viabilizado simular problemas de resolução complexa como sistemas reacionais, sistemas multifásicos, perfis aerodinâmicos, etc. Como exemplo de simulações CFD temos: a fluidodinâmica do leito de jorro (Duarte et al., 2005; Santos, 2008; Vieira Neto et al., 2008; Santos, 2011); a separação sólido-líquido em hidrociclones convencionais e filtrantes (Vieira, 2006); escoamento laminar de fluidos não Newtonianos em seções anulares (Vieira Neto, 2011), dentre outros. O estudo da fluidodinâmica de equipamento de separação sólido-fluido é um exemplo de modelagem multifásica que envolve uma fase fluida e uma fase particulada.

Este trabalho tem como objetivo principal simular alguns estudos de caso sobre separação sólido-fluido em câmaras de separação gravitacional, que serão usados como material didático ilustrativo nas aulas de Operações Unitárias I. Serão simuladas as condições de escoamento baseadas em exercícios ministrados em sala de aula, retirado dos principais livros de sistemas particulados. Para tal, serão empregadas simulações CFD, que possibilitarão aos alunos visualizar o perfil de velocidades das partículas no interior do equipamento através de figuras e vídeos, facilitando a compreensão dos fenômenos envolvidos na coleta de partículas dentro do separador. Este estudo é fruto de um treinamento para alunos do Programa Jovens Talentos, em que os alunos estudam separadamente a técnica de CFD aplicada a diversos equipamentos.

\section{ESTUDOS DE CASO}

A separação sólido-fluido em câmaras de separação gravitacional é um método baseado na sedimentação livre, considerando o próprio peso e a velocidade terminal das partículas. Estas câmaras apresentam área transversal suficientemente grande através da qual, o fluido passa com baixa velocidade, dando tempo para que partículas possam sedimentar no fundo ou na base da câmara. A eficiência de coleta pode ser melhorada incluindo-se chicanas ou telas, permitindo o aumento da velocidade do fluido e a maior retenção das partículas. Este é o tipo mais simples para coleta da partículas dispersas em um fluido (líquido ou gás). O dimensionamento do equipamento é feito para operar com baixas velocidades de fluido $(0,02 \mathrm{~m} / \mathrm{s}$ até $4,0 \mathrm{~m} / \mathrm{s})$, para evitar a redispersão das partículas. Se não houver turbulência, as partículas decantarão com velocidade $v_{t}$. O tempo de queda das partículas $(t)$ em função da velocidade terminal $\left(v_{t}\right)$ é dado pela razão entre a altura do tanque $\mathrm{H}$ e $v_{t}$. Assim, temos que:

$$
d_{p}=\frac{\operatorname{Re} \mu}{\rho v_{t}}
$$




$$
\begin{aligned}
& v_{t}=\frac{Q}{B L} \\
& \frac{C_{D}}{\operatorname{Re}}=\frac{4}{3} \frac{\left(\rho_{\text {part }}-\rho\right) \mu g}{\rho_{f}{ }^{2} v_{t}^{3}}
\end{aligned}
$$

sendo $d_{p}$ o diâmetro de partícula que é coletada no equipamento, Re o número de Reynolds da fase particulada; $\rho_{\text {part }}$ e $\rho$ as densidades da partícula e do fluido, respectivamente; $B$ o comprimento do tanque; L é a largura do tanque; $C_{D}$ é o coeficiente de arraste; $\mu$ é a viscosidade do fluido e $g$ a força gravitacional.

Segundo Cremasco (2012), identificando o termo BHL como sendo o volume V da câmara, o diâmetro da menor partícula retida na câmara (diâmetro de corte) pode então ser definido como:

$$
d_{p}=\left[\frac{18 \mu}{K_{1}\left(\rho_{p}-\rho\right) g}\left(\frac{H Q}{V}\right)\right]^{\frac{1}{2}}
$$

Para a separação sólido-líquido, o equipamento é conhecido como tanque de areia, enquanto que para a separação sólido-gás, o equipamento é chamado de câmara de poeira.

\subsection{Caso 1: Câmara de Poeira}

A câmara de poeira é uma Operação Unitária que tem por objetivo a separação sólido-gás, no campo gravitacional, para retirar e classificar poeiras de correntes gasosas é um método baseado na sedimentação livre considerando o próprio peso e a velocidade terminal das partículas. Este caso consiste na simulação do Exemplo 8.1, resolvido em Cremasco (2012). A câmara de poeira opera em três compartimentos. Deseja-se estimar a faixa granulométrica das partículas retidas em cada compartimento, sabendo que a vazão de ar é igual $140 \mathrm{~m}^{3} / \mathrm{min}$. A massa específica das partículas é de $3000 \mathrm{~kg} / \mathrm{m}^{3}$ e a esfericidade é 0,75 . A corrente de ar está a $20{ }^{\circ} \mathrm{C}(\rho=1,091$ $\mathrm{kg} / \mathrm{m}^{3}$ e $v=1,7510^{-5} \mathrm{~m}^{2} / \mathrm{s}$ ).

\subsection{Caso 2: Tanque de areia}

O problema proposto consiste em estudar a separação das partículas provenientes de uma suspensão diluída de cal em água contendo areia como produto indesejável. As partículas de cal tem densidade de $2400 \mathrm{~kg} / \mathrm{m}^{3}$ e esfericidade e as de areia tem densidade de $2650 \mathrm{~kg} / \mathrm{m}^{3}$. O tanque de areia estudado tem comprimento $\mathrm{L}=1 \mathrm{~m}$, largura $\mathrm{B}=0,5 \mathrm{~m}$ e altura $\mathrm{H}=0,05 \mathrm{~m}$, e opera com água à $20^{\circ} \mathrm{C}\left(\rho=1000 \mathrm{~kg} / \mathrm{m}^{3}\right.$ e $\left.\mu=1 \mathrm{cP}\right)$. Na simulação deste caso, supôs-se tratar de partículas esféricas. Em um estudo posterior, será avaliado o efeito da esfericidade das partículas sobre a eficiência de coleta.

Nesse exercício, deve-se calcular a faixa de diâmetro de partículas de areia e cal que são coletadas no equipamento para a velocidade de alimentação de fluido de $0,5 \mathrm{~m} / \mathrm{s}$, a fim de construir a grade de eficiência individual de coleta que mostra qual a porcentagem de partículas de um dado tamanho que são coletadas no equipamento. Com esses dados, é possível propor um caso de separação da cal a partir de uma corrente aquosa de cal contaminada com areia. 


\section{METODOLOGIA}

Para cada caso estudado, foram definidas: a velocidade inicial do fluido, a geometria do equipamento e as propriedades do fluido (densidade, temperatura, viscosidade) e das partículas presentes na suspensão (concentração volumétrica, diâmetro, densidade, esfericidade). As condições de contorno foram adequadamente definidas: nas paredes, a velocidade e concentração de fluido são nulas e nas paredes inferiores do equipamento as partículas são coletadas; na entrada define-se a velocidade do fluido de cada simulação; na saída, define-se a pressão como sendo atmosférica e permite-se a que as partículas deixem o equipamento.

Para tal, a malha computacional correspondente a geometria de cada caso foi confeccionada, em 2 D, empregando-se o "software" GAMBIT, que disponibiliza uma interface com o "software" de fluidodinâmica computacional FLUENT ${ }^{\circledR}$. A Figura 1 apresenta as malhas computacionais utilizadas para o Caso 1 (Figura 1a), com 5400 elementos quadrangulares e 2388 elementos triangulares, e para o Caso 2 (Figura 1b), com 40800 elementos quadrangulares.

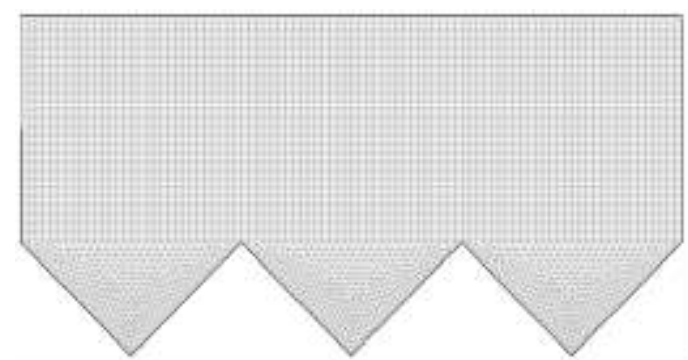

(a)

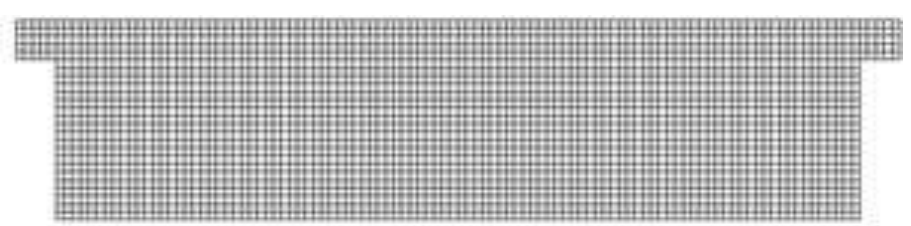

(b)

Figura 1 - Malha computacional usada nas simulações: (a) câmara de poeira; (b) tanque de areia.

O perfil de velocidade do fluido na câmara é obtido para o regime permanente. Como a fração volumétrica de sólidos na suspensão é baixa, as interações partícula-partícula podem ser desprezadas, e assim, pode-se utilizar o modelo de Fase Discreta, em que a equação do movimento da partícula é resolvida considerando o campo de velocidades da vizinhança, considerando apenas a interação da partícula com o fluido. Deste modo, é possível obter as trajetórias de cada partícula no equipamento, bem como a quantidade de partículas que não são coletadas, ou seja, que deixam a corrente de saída do equipamento.

Assim, as equações da continuidade e movimento para o fluido, e subsequentemente a do movimento das partículas discretas, são então discretizadas pelo método de Volumes Finitos, utilizado pela maioria dos softwares comerciais de CFD, pois garante resultados coerentes mesmo utilizando malhas grosseiras e não estruturadas (Santos, 2008).

A seguir tem-se uma descrição do Modelo de Fase Discreta, detalhado em Silva (2012).

\subsection{Modelo Matemático de Fase Discreta}

Há duas maneiras de abordar um problema quando se trata de cálculo do movimento de partículas num fluido em escoamento utilizando o vetor de velocidades $\mathrm{v}(\mathrm{x}, \mathrm{y}, \mathrm{z}, \mathrm{t})$. A visão Lagrangeana fixa coordenadas fixas, $\mathrm{x}_{1}, \mathrm{y}_{1} \mathrm{e} \mathrm{z}_{1}$ nas funções de campo de velocidade e assim, podemos medir a velocidade a velocidade das partículas que passam por este ponto a cada instante e o vetor torna-se $\left(\mathrm{x}_{1}, \mathrm{y}_{1}, \mathrm{z}_{1}, \mathrm{t}\right)$. 
Para a descrição da trajetória das partículas no tanque de areia, pode-se utilizar o modelo de Fase discreta, recomendado para condições de suspensões diluídas, ou seja, com uma porcentagem volumétrica de sólidos em até $10 \%$. Nesta abordagem, admite-se que a baixa quantidade de partículas não influencia o escoamento do fluido, e assim este pode ser obtido separadamente, como se escoasse sozinho (Silva, 2012).

O campo de velocidades do fluido é obtido pela resolução da equação da continuidade e da equação de quantidade de movimento para a fase fluida:

$$
\begin{aligned}
& \frac{\partial}{\partial t}(\rho)+\nabla(\rho \vec{v})=S_{m} \\
& \frac{\partial}{\partial t}(\rho \vec{v})+\nabla \cdot(\rho \vec{v} \vec{v})=-\nabla p+\nabla \cdot \overline{\bar{\tau}}+\rho \vec{g}+\vec{F}
\end{aligned}
$$

onde $S_{m}$ representa a massa adicionada para a fase contínua (fluida) por causa da dispersão da fase dispersa (partículas); - $\nabla$ p é gradiente de pressão estática; $\rho \vec{g}$ representa a força gravitacional e $\vec{F}$ as forças de corpo, $\overline{\bar{\tau}}$ é tensor tensão de deformação da fluida, que pode ser descrito por:

$$
\overline{\bar{\tau}}=\mu\left[\left(\nabla \vec{v}+\nabla \vec{v}^{T}\right)-\frac{2}{3} \nabla \cdot \vec{v} I\right]
$$

onde $\mu$ é a viscosidade do fluido; é o tensor unitário e o termo $2 / 3 \nabla \cdot \vec{v} I$ está relacionado ao efeito da dilação de volume.

Após a simulação da distribuição de velocidade do fluido no interior do equipamento, as partículas são injetadas, ou seja, a trajetória das partículas pode ser predita através da integração da equação do movimento da partícula, a qual contabiliza o balanço entre as principais forças que atuam sobre a partícula, conforme a equação:

$$
\frac{\partial u_{p}}{\partial t}=F_{D}\left(u-u_{p}\right)+g_{z}\left(\frac{\rho_{p}-\rho}{\rho_{p}}\right)+F_{z}
$$

onde $u$ é a velocidade do fluido, $u_{p}$ é a velocidade da partícula, $\rho_{p}$ e $\rho$ são a densidade da partícula e do fluido, respectivamente. As demais forças que podem atuar sobre a partícula são representadas por $F_{z}$.

O termo $F_{D}\left(u-u_{p}\right)$ representa a força de arraste por unidade de massa da partícula e pode ser representado por:

$$
F_{D}=\frac{18 \mu}{\rho_{p} d_{p}^{2}} \frac{C_{D} \mathrm{Re}}{24}
$$

\section{RESULTADOS E DISCUSSÕES}

\subsection{Caso 1 - Câmara de poeira}

A Figura 2 mostra a trajetória de partículas, coloridas por diâmetro $[\mu \mathrm{m}]$, para velocidade do gás de $0,777 \mathrm{~m} / \mathrm{s}$, que corresponde à vazão de ar do exemplo reproduzido do livro. 


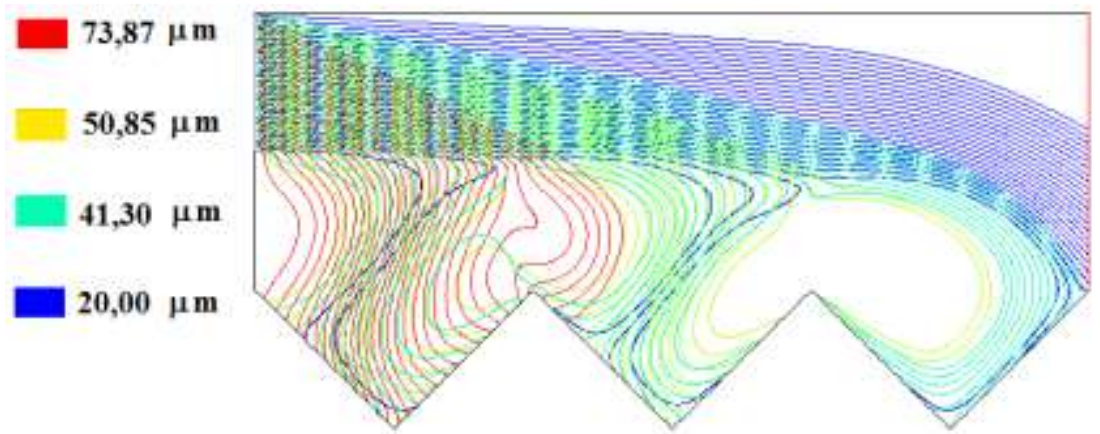

Figura 2 - Trajetórias de partículas, coloridas por diâmetro [ $\mu \mathrm{m}]$, no interior da câmara de poeira.

Verificou-se, conforme o exercício do livro, que as partículas maiores que 73,87 $\mu \mathrm{m}$ ficam retidas na $1^{\text {a }}$ câmara, enquanto que as partículas acima de 50,85 $\mu \mathrm{m}$ ficam retidas na $2^{\mathrm{a}}$ câmara, e que na $3^{\text {a }}$ câmara ficam as partículas acima de $41,30 \mu \mathrm{m}$. Já as partículas menores (como as de $20 \mu \mathrm{m})$ saem juntamente com a corrente de gás na saída do equipamento. Observa-se também uma recirculação das partículas menores dentro do equipamento, uma vez que, elas podem ser arrastadas juntamente com as outras partículas. A Figura 2 é um ótimo material de apoio em sala de aula, pois, permite que os alunos visualizem a separação das partículas de diferentes tamanhos ao longo dos compartimentos da câmara de poeira.

\subsection{Caso 2 - Tanque de Areia}

Após a construção da malha computacional e a definição do problema no software de simulação, foi simulado inicialmente apenas o escoamento do fluido, a água, para as três velocidades de alimentação propostas. Depois da obtenção do perfil de velocidade do fluido, foram injetadas partículas de cal e de areia de diferentes tamanhos, a fim de construir o gráfico da eficiência individual de coleta das partículas de diferentes diâmetros, apresentado na Figura 3. Pode-se observar que, tanto para areia quanto para a cal, as partículas de menor diâmetro são coletadas com baixa eficiência, sendo que à medida que o diâmetro das partículas aumenta, aumenta-se a eficiência de coleta. Comparando as grades de eficiência de cal e de areia, de acordo com a Figura 3, observa-se que a faixa diâmetro de coleta das partículas é muito parecida. Assim, a separação completa da cal só será possível se as partículas de areia presentes na mistura apresentar diâmetros bem maiores que os das partículas de calcário.

A Figura 4 mostra a trajetória das partículas dentro do equipamento, coloridas de acordo com o diâmetro das partículas. Observa-se que partículas maiores são coletadas no início do equipamento, próximas à entrada, enquanto as partículas menores saem na saída do equipamento. Isso ocorre por que as partículas maiores são mais pesadas e sentem mais o campo gravitacional.

Como exemplo de separação sólido-sólido, suponha que uma corrente de suspensão aquosa de partículas de cal, com faixa de diâmetro de $10 \mu \mathrm{m}$ a $200 \mu \mathrm{m}$, está contaminada com partículas de areia $\left(200 \mu \mathrm{m}<\mathrm{d}_{\mathrm{p}}<400 \mu \mathrm{m}\right)$ que são indesejáveis no processo e, portanto, devem ser removidas usando o tanque areia com a geometria citada neste trabalho, operando a uma velocidade de alimentação de fluido de $0,5 \mathrm{~m} / \mathrm{s}$ na entrada. Nesta velocidade de fluido de $0,5 \mathrm{~m} / \mathrm{s}$, o diâmetro de corte de partículas de areia é de $200 \mu \mathrm{m}$. Assim, todas as partículas de areia são coletadas, enquanto apenas uma quantidade de cal é retida no tanque. O importante é que a corrente de cal que deixa o equipamento sai isenta de areia, como mostra a simulação CFD da Figura 5. 


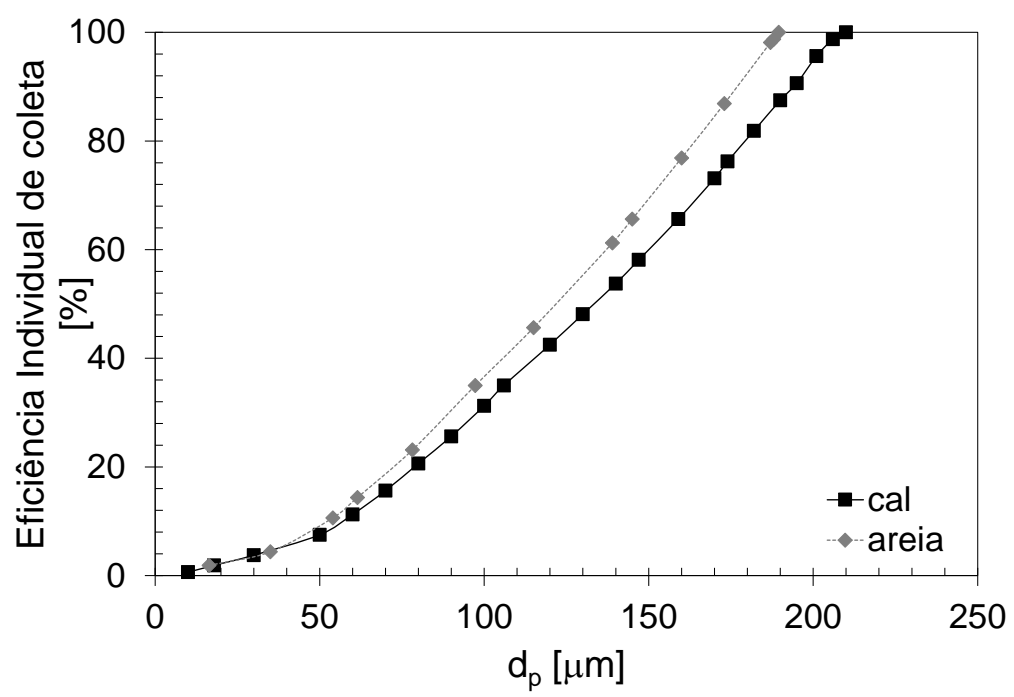

Figura 3 - Grades de eficiência em função do diâmetro de coleta das partículas de cal e areia, para velocidade de alimentação de água de $0,5 \mathrm{~m} / \mathrm{s}$.

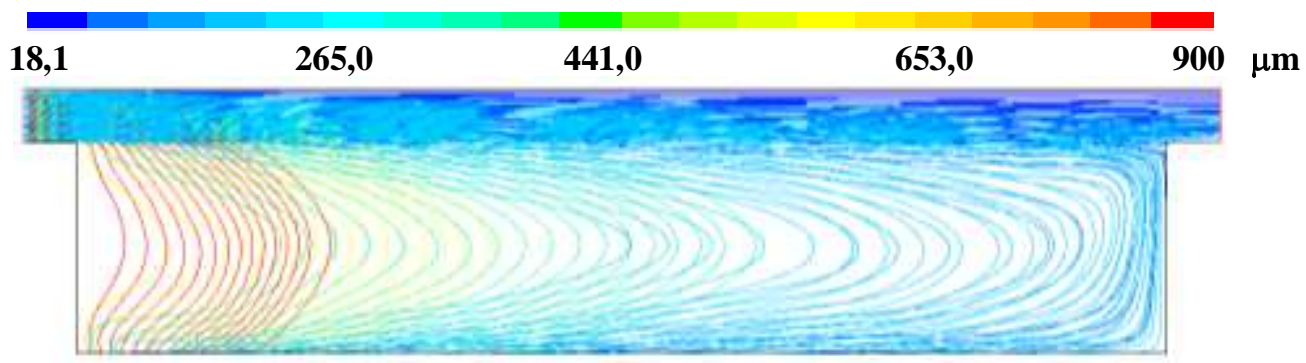

Figura 4 - Trajetória das partículas de cal coloridas por diâmetro $[\mu \mathrm{m}]$, para a velocidade de alimentação de líquido de $0,5 \mathrm{~m} / \mathrm{s}$

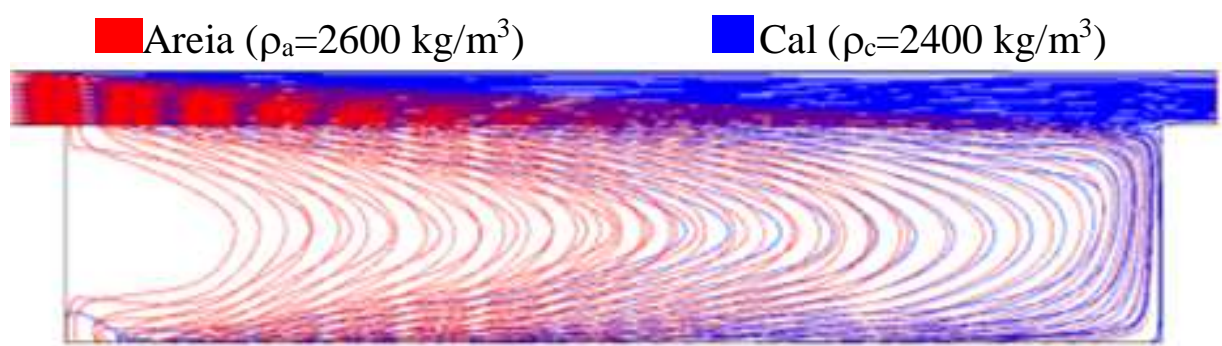

Figura 5 - Exemplo do uso do tanque de areia para separação de partículas de cal $\left(10 \mu \mathrm{m}<\mathrm{d}_{\mathrm{p}}<200 \mu \mathrm{m}\right)$ e areia $\left(200 \mu \mathrm{m}<\mathrm{d}_{\mathrm{p}}<400 \mu \mathrm{m}\right)$

As Figuras 4 e 5 são excelentes como material de apoio em sala de aula. É possível verificar a trajetória das partículas de acordo com seu diâmetro e plotar a trajetória colorida pela densidade das partículas, deixando claro ao aluno que na corrente que deixa o equipamento contém apenas partículas de cal. Essas imagens podem ajudar os alunos a compreender o funcionamento do equipamento e sua utilidade na área de separação de misturas envolvendo material particulado. Há ainda a possibilidade de apresentar em sala de aula um vídeo que mostra a trajetória das partículas der uma forma mais dinâmica. 


\section{CONCLUSÕES}

Através das simulações CFD foi possível obter figuras de contornos de velocidade do fluido, bem como, de trajetórias de partículas de diferentes tamanhos. Estas figuras permitiram compreender a dinâmica dos fluidos e das partículas no interior dos equipamentos de separação sólido-fluido em campo gravitacional. Foi possível verificar a quantidade de partículas coletadas pela câmara de poeira e pelo tanque de areia, o que permite obter a eficiência global de separação de cada equipamento. Quando a suspensão em estudo se tratar de uma mistura de partículas de diferentes materiais, é possível calcular a concentração da corrente de saída para cada material, bem como, a eficiência de separação individual para cada faixa de tamanho de partículas.

\section{AGRADECIMENTOS}

Os autores agradecem ao $\mathrm{CNPq}$ pela bolsa do Programa Jovens Talentos e à Universidade Federal do Triângulo Mineiro e à FAPEMIG (PCE-00201-14).

\section{REFERÊNCIAS}

CREMASCO, M.A. Operações Unitárias em Sistemas Particulados e Fluidomecânicos. São Paulo: Editora Blücher, 2012.

DUARTE, C.R.; MURATA, V.V.; BARROZO, M.A.S. A Study of the Fluid Dynamics of the Spouted Bed Using CFD. Brazilian Journal of Chemical Engineering, v. 22, p. 263-270, 2005.

MASSARANI, G. Fluidodinâmica em Sistemas Particulados. Rio de Janeiro: Universidade Federal do Rio de Janeiro, 1997.

SANTOS, K.G. Aspectos fundamentais da pirólise de biomassa em leito de jorro: Cinética e fluidodinâmica do processo. Tese de Doutorado, Faculdade de Engenharia Química da Universidade Federal de Uberlândia, Uberlândia, 2011.

SANTOS, K.G.; MURATA, V.V.; BARROZO, M.A.S. Three-dimensional computational fluid dynamics modeling of spouted bed, Can. J. Chem. Eng., v. 87, p.211-219, 2009.

SILVA, D.O. Otimização da separação sólido líquido em hidrociclones mediante modificações geométricas, Tese de Doutorado, Programa de Pós-graduação em Engenharia Química da Universidade Federal de Uberlândia, Uberlândia, 2012.

VIEIRA, L.G.M. Otimização dos processos de separação em hidrociclones filtrantes. Tese de Doutorado, Faculdade de Engenharia Química da Universidade Federal de Uberlândia, Uberlândia, 2006.

VIEIRA NETO, J.L.; DUARTE, C.R.; MURATA, V.V.; BARROZO, M.A.S. Effect of a Draft Tube on the Fluid Dynamics of a Spouted Bed: Experimental and CFD Studies. Drying Technol., v. 26, p. 299-307, 2008. 